\title{
The effectiveness and cost-effectiveness of inpatient specialist palliative care in acute hospitals for adults with advanced illness and their caregivers (Protocol)
}

\author{
Daveson BA, Smith M, Yi D, McCrone P, Grande G, Todd C, Gysels M, Costantini M, \\ Murtagh FE, Higginson IJ, Evans CJ
}

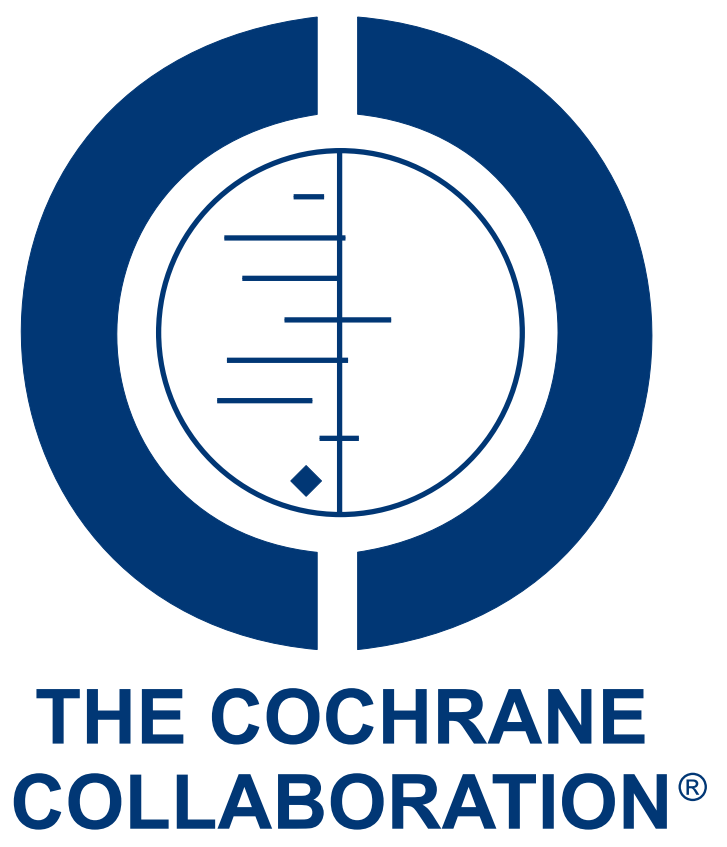

This is a reprint of a Cochrane protocol, prepared and maintained by The Cochrane Collaboration and published in The Cochrane Library 2015, Issue 3

http://www.thecochranelibrary.com

\section{WILEY}

The effectiveness and cost-effectiveness of inpatient specialist palliative care in acute hospitals for adults with advanced illness and their caregivers (Protocol)

Copyright $\odot 2015$ The Cochrane Collaboration. Published by John Wiley \& Sons, Ltd. 
TABLE OF CONTENTS

HEADER . . . . . . . . . . . . . . . . . . . . . . . . . . . . . . . . . . . . . . . 1

ABSTRACT . . . . . . . . . . . . . . . . . . . . . . . . . . . . . . . . . . . . . . . . .

BACKGROUND . . . . . . . . . . . . . . . . . . . . . . . . . . . . . . . . . . . . . .

OBJECTIVES . . . . . . . . . . . . . . . . . . . . . . . . . . . . . . . . . . . . . . . . . .

METHODS . . . . . . . . . . . . . . . . . . . . . . . . . . . . . . . . . . . . . . 44

ACKNOWLEDGEMENTS . . . . . . . . . . . . . . . . . . . . . . . . . . . . . . . . . . . . . . . . . . . .

REFERENCES . . . . . . . . . . . . . . . . . . . . . . . . . . . . . . . . . . . . . . 10

APPENDICES . . . . . . . . . . . . . . . . . . . . . . . . . . . . . . . . . . . . . 13

CONTRIBUTIONS OF AUTHORS . . . . . . . . . . . . . . . . . . . . . . . . . . . . . . . . . . . . . . .

DECLARATIONS OF INTEREST . . . . . . . . . . . . . . . . . . . . . . . . . . . . . . . 15

SOURCES OF SUPPORT . . . . . . . . . . . . . . . . . . . . . . . . . . . . . . . . . .

The effectiveness and cost-effectiveness of inpatient specialist palliative care in acute hospitals for adults with advanced illness and their i caregivers (Protocol)

Copyright $\odot 2015$ The Cochrane Collaboration. Published by John Wiley \& Sons, Ltd. 


\title{
The effectiveness and cost-effectiveness of inpatient specialist palliative care in acute hospitals for adults with advanced illness and their caregivers
}

\author{
Barbara A Daveson ${ }^{1}$, Melinda Smith ${ }^{1}$, Deokhee $\mathrm{Yi}^{1}$, Paul McCrone ${ }^{2}$, Gunn Grande ${ }^{3}$, Chris Todd $^{3}$, Marjolein Gysels ${ }^{4}$, Massimo \\ Costantini $^{5}$, F E Murtagh ${ }^{1}$, Irene J Higginson ${ }^{1}$, Catherine J Evans ${ }^{1}$
${ }^{1}$ Department of Palliative Care, Policy and Rehabilitation, Cicely Saunders Institute, King's College London, London, UK. ${ }^{2}$ Institute of Psychiatry, King's College London, London, UK. ${ }^{3}$ School of Nursing, Midwifery and Social Work, University of Manchester, Manchester, UK. ${ }^{4}$ Amsterdam Institute of Social Science Research, University of Amsterdam, Amsterdam, Netherlands. ${ }^{5}$ Palliative Care Unit, IRCCS Arcispedale S. Maria Nuova, Reggio Emilia, Italy

Contact address: Barbara A Daveson, Department of Palliative Care, Policy and Rehabilitation, Cicely Saunders Institute, King's College London, London, UK. barbara.daveson@kcl.ac.uk.

Editorial group: Cochrane Pain, Palliative and Supportive Care Group.

Publication status and date: New, published in Issue 3, 2015.

Citation: Daveson BA, Smith M, Yi D, McCrone P, Grande G, Todd C, Gysels M, Costantini M, Murtagh FE, Higginson IJ, Evans CJ. The effectiveness and cost-effectiveness of inpatient specialist palliative care in acute hospitals for adults with advanced illness and their caregivers. Cochrane Database of Systematic Reviews 2015, Issue 3. Art. No.: CD011619. DOI: 10.1002/14651858.CD011619.

Copyright (C) 2015 The Cochrane Collaboration. Published by John Wiley \& Sons, Ltd.

\begin{abstract}
A B S T R A C T
This is the protocol for a review and there is no abstract. The objectives are as follows:

To assess the effectiveness and cost-effectiveness of inpatient specialist palliative care in acute hospitals for adults with advanced illness and their unpaid caregivers.
\end{abstract}

\section{B A C K G R O U N D}

The global burden of disease has increased due to global demography of lowered fertility, increased longevity, and reduced childhood and infant infectious disease mortality. This change is placing considerable strain on healthcare systems internationally (Murray 2012). Most adults develop one or more chronic illnesses with which they may live for many years before they die. For a minority of patients with serious illness (for example, metastatic colon cancer), the time following diagnosis is characterised by a stable period of relatively good functional and cognitive performance, followed by a predictable and short period of functional and clinical decline. However, for most patients with serious illness (for ex- ample, heart or lung disease, Parkinson's disease, dementia, stroke, neuro-muscular degenerative diseases and many cancers), the time following diagnosis is characterised by months to years of physical and psychological symptom distress, progressive functional dependence and frailty, considerable family support needs and high healthcare resource use (Murray 2005). In addition to increased clinical complexity, the rise of ageing populations has led to considerable healthcare costs globally. This has occurred despite efforts to reduce acute hospital care expenditure in many high-income countries, including, for example, in the United States (Kashihara 2012), the United Kingdom (Lafond 2014), and Australia (AIHW 2014).

The effectiveness and cost-effectiveness of inpatient specialist palliative care in acute hospitals for adults with advanced illness and their caregivers (Protocol)

Copyright $\odot 2015$ The Cochrane Collaboration. Published by John Wiley \& Sons, Ltd. 
It could be argued that increased staffing costs and the introduction or expansion of novel services in hospitals, such as specialist palliative care, plays a role in this increased expenditure. For example, in the United States, over the past 12 years, palliative care prevalence in hospitals with 50 or more beds has increased 164\%, to $61 \%$ of hospitals (Center to Advance Palliative Care 2014). Furthermore, the growth of specialist palliative care in acute hospitals is likely to continue in the foreseeable future as most older adults ( $\geq 65$ years old) die in hospitals ( $71 \%$ of all hospital deaths in the United States) (Zhao 2010), the majority of deaths in hospital occur due to terminal illness (Gruneir 2007), and also because deaths in institutional care persist into older stages of life, with one in five centenarians dying in hospital (Evans 2014).

However, perhaps specialist palliative care is part of the solution rather than part of the problem. Evidence shows that specialist palliative care improves clinical outcomes and quality of care (Higginson 2003), can reduce hospital costs (Higginson 2003; Morrison 2008), and help contain costs within the last year of life (Hatziandreu 2008). Reduced hospitalisation rates, invasive procedures and intensive care admissions have also been found in those receiving hospice care versus matched subjects who died without hospice care (Obermeyer 2014). Furthermore, specialist palliative care, which includes bereavement care and preparatory grief work, has the potential to help unpaid caregivers access the care they need related to the death of a loved one (DoH 2008). Data shows that those with prolonged grief disorder may underutilise services that may help them (Lichtenthal 2011). This means that specialist palliative care may even help with preventing medical problems for those at risk of developing complicated or prolonged grief. Earlier access to specialist palliative care has been shown to improve both patient and unpaid caregiver outcomes (Higginson 2011; Temel 2010; Zimmerman 2014).

This Cochrane systematic review will assess the effectiveness and cost-effectiveness of specialist palliative care for adult patients with advanced illness receiving care in acute hospitals and for their unpaid caregivers. The review findings have the potential to aid the future development, funding and implementation of evidencebased inpatient specialist palliative care. This may help transform services, which have mostly developed locally in culturally responsive ways in relation to local needs and populations (Higginson 2003; Kamal 2013). Therefore, the review will help deliver specialist palliative care services in the midst of increased ageing populations that present with complex clinical needs against a backdrop of fiscal constraint and increased healthcare utilisation.

\section{Description of the condition}

At the heart of palliative care is the belief that every person is unique, autonomous, and that they have the right to continue to live and enjoy quality of life even though they are diagnosed with an advanced, life-limiting or life-threatening illness. Some of the underlying principles that underscore palliative care, such as individuality, autonomy and self-determination, although recognised in the field of medicine, are sometimes difficult to deliver in environments, including acute hospital settings, which are typically focused on disease-modifying and curative treatments. Plus, the need for coordinated care for those at the end of life is not always delivered and this can result in increased hospitalisations and suboptimal clinical outcomes (Higginson 2003; Walsh 2011). Poor coordination is a problem especially evident for vulnerable groups, including older adults (Smith 2012). It is a problem that can be improved through specialist palliative care input (Higginson 2003). Although increasingly recognised internationally as essential to healthcare, only 1 in 10 who needs palliative care receives it (WPCA/WHO 2014). This is despite palliative care being shown to improve clinical outcomes, patient-centred decision-making and care coordination, and reduce hospital costs through significant reductions in pharmaceutical, laboratory and intensive care unit costs (Higginson 2003; Morrison 2008; Temel 2010; Zimmerman 2014). Palliative care therefore remains on the margins of mainstream medicine, despite its growth in inpatient specialist palliative care services and an increasing evidence base outlining clinical and fiscal benefits (WPCA/WHO 2014). This issue potentially places patients and their unpaid caregivers at risk of receiving care that focuses on disease-modification at the expense of optimal outcomes, holistic care and efficiency.

\section{Description of the intervention}

The intervention examined in this review is inpatient specialist palliative care. Inpatient specialist palliative care encompasses interventions delivered to patients with advanced (Coalition to Transform Advanced Care 2013), life-limiting (Palliative Care Australia 2005), or life-threatening illness (NCP 2013), which is likely to compromise their quality of life (The WHOQOL Group 1995). The care is provided to the patient while they are admitted as inpatients to acute care hospitals. The intervention aims to prevent and/or relieve physical, psychological, social and spiritual problems. It is provided to patients with a malignant and/or nonmalignant condition who may or may not be at the end of their life (NIH 2004). Population-based estimates of specialist palliative care have indicated which populations require specialist palliative care (Murtagh 2014), including those with malignant neoplasms and non-malignant and other health-related conditions, specifically: heart disease, including cerebrovascular disease, renal disease, liver disease, respiratory disease, neurodegenerative disease (Huntington's disease, Parkinson's disease, multiple sclerosis, motor neuron disease, multi-system degeneration, progressive supranuclear ophathlmoplegia, Alzheimer's dementia and senility), and/or h uman immunodeficiency virus (HIV) infection/acquired immune deficiency syndrome (AIDS).

Inpatient specialist palliative care comprises of the following essential components: 
1. care coordinated by a multi-professional or multidisciplinary team;

2. collaboration between specialist palliative care providers and generalist providers;

3. holistic care; and

4. complexity, feelings of loss and uncertainty (NCP 2013).

Specialist palliative care is differentiated from generalist palliative care. Specialists are likely to have received higher specialist training in palliative care work and services focus mainly/exclusively on patients with palliative care needs, whereas for generalists, provision of palliative care is a component of their service provision (Shipman 2008). Specialist care is mostly provided to patients with advanced, life-limiting and / or life-threatening illness who present with complex needs (Palliative Care Australia 2005). Complexity, although sometimes difficult to define, involves clinical complexity and its interaction with the confidence and / or ability of the lead clinical team (generalists) to address the presenting need. Complexity may stem from underlying pathological (disease) process, ethical complexity or both. Complexity usually involves intertwined and multiple factors, related to age, the serious nature of illness, social or familial backgrounds, and/or the nature of a symptom (for example, the usualness or intractable nature of the symptom) (Palliative Care Australia 2005; Quill 2013).

The intervention is provided to patients who are inpatients in an acute hospital and their families. Inpatient wards include, for example, palliative care units in the hospital, intensive care units, oncology wards, care of the elderly wards, or accident and emergency departments. Specialist palliative care provided to unpaid caregivers in hospital settings and/or outpatients will be included. This is because unpaid caregivers are likely to be seen as outpatients or in treatment rooms by hospital staff in the hospital in order to address pre-bereavement needs. The intervention is administered by hospital staff who have completed specialist training in palliative care or who have obtained clinical competencies and professional characteristics required for the delivery of inpatient specialist palliative care through clinical experience working (NCPC 2012).

Some inpatient specialist palliative care services involve pre-bereavement interventions to help prepare the unpaid caregiver for the death of their loved one, however not all services include this additional intervention (Bodenbach 2005; Field 2004; Reid 2006). Pre-bereavement interventions are inpatient specialist palliative care interventions administered to prevent or manage bereavement-related physical, psychological, social and spiritual problems experienced by unpaid caregivers prior to the death of the patient. Inpatient specialist palliative care interventions that include pre-bereavement interventions either to the unpaid caregiver alone or together with the patient will be included in this review.

\section{How the intervention might work}

Although positive outcomes, such as symptom reduction, improved quality of care and care coordination, and reduced hospital costs can result from specialist palliative care, qualitative modelling and empirical testing is yet to definitively establish how inpatient specialist palliative care might work. Therefore, any descriptions of how specialist palliative care may work are speculative. That acknowledged, inpatient specialist palliative care may work with patients by the following :

- directly improving symptoms (including physical and/or psychological symptoms, such as uncertainty and feelings of loss) through specialist interventions and holistic care (Temel 2010);

- improving care quality and the tenor of care through assisting patients, unpaid caregivers and staff through delivering or facilitating improved care coordination and person-centred holistic care (Daveson 2014a; Pinnock 2011);

- reducing futile medical interventions by mitigating against disease-modifying priorities while also enabling patient dignity and autonomy (Harris 2013);

- reducing unnecessary hospital costs through significant reduction in pharmaceutical, laboratory and intensive care unit costs (Morrison 2008).

Findings from a published systematic review indicated that the intervention may work for caregivers prior to the death of the patient through emphasising the positive aspects of caregiving by providing relevant information, guidance and instruction; improving the caregiver's understanding of their experiences and role to result in increased caregiving competencies and knowledge; aiding their interpretation of their circumstance and normalising their emotional responses to caregiving demands; and/or enabling their involvement in care planning, where possible (Harding 2012; Hudson 2005). Engaging both patients and caregivers in life review within consultations may work to reduce caregivers' stress (Allen 2008). The intervention may also work by providing caregivers with individual support to see problems differently, draw out their optimism, helping them to plan and by providing them with access to expert information. This has been shown to improve their quality of life overall while also decreasing caregiver burden and tasks (McMillan 2006). Specialist palliative care may also ensure timely assessment of needs, adaptive coping and access to needs-based care through pre-bereavement work (Lichtenthal 2011). The intervention may therefore also work via a preventive mechanism.

\section{Why it is important to do this review}

This systematic review is important to complete due to two reasons. First, there is a growing body of evidence that shows that aggressive and, at times, futile treatments are being implemented with patients in acute hospital settings during the end of life (Ho 2011). These treatments can correspond with negative financial, clinical and utilisation outcomes (Sullivan 2011), and may not always reflect patient preferences (Daveson 2014b). Specialist pal-

The effectiveness and cost-effectiveness of inpatient specialist palliative care in acute hospitals for adults with advanced illness and their 
liative care has been shown to both improve clinical outcomes and reduce hospital costs (compared to usual care), thus examining the evidence in this review is important as it may help improve care while also reducing hospital costs. Second, the numbers of inpatient specialist palliative care teams are increasing (Higginson 2003; Meier 2011). This is occurring in response to unmet palliative needs of inpatients and their unpaid caregivers (Meier 2011), yet clarity regarding the effective components of the intervention remains unknown. This review is therefore important as it can assist with providing much-needed solutions to problems, and clarity regarding the effectiveness and cost-effectiveness of the component parts of specialist palliative care. In essence, the review may be helpful in addressing some of the problems encountered by contemporary healthcare systems and services, service-users, clinicians, policy makers, researchers and commissioners.

\section{O B J E C T I V E S}

To assess the effectiveness and cost-effectiveness of inpatient specialist palliative care in acute hospitals for adults with advanced illness and their unpaid caregivers.

\section{METHODS}

\section{Criteria for considering studies for this review}

\section{Types of studies}

We will examine both effectiveness and cost-effectiveness components. Although the number of randomised controlled trials (RCTs) in palliative and end-of-life care is steadily increasing (Rinck 1997), they remain few in number. Non-randomised studies can provide important understanding on the effectiveness of palliative care services (Higginson 2003), but only with careful attention paid to the likelihood of bias (Field 2004; Strobe 2001). We will include studies that examine inpatient specialist palliative care through an RCT or a controlled clinical trial (CCT). Individual- and cluster-unit randomisation will also be included. The type of non-randomised studies we are interested in include: quasi-experimental studies, interrupted time series (ITS) studies, controlled before and after (CBA) studies, cohort and case-control studies. We will use the list of study design features given in the Cochrane Handbook for Systematic Reviews of Interventions to identify the characteristics of non-randomised studies in order to include all eligible studies (Higgins 2011).

All studies must evaluate effectiveness regarding one of the stated primary and/or secondary outcomes stipulated for this review. In the economic component of the review, studies to be included are those that are conducted alongside (or as part of) the main effectiveness trial and ones that also meet the eligibility criteria for the effectiveness component. Full economic evaluation (i.e. costeffectiveness analyses, cost-utility analyses, cost-benefit analyses); partial economic evaluations (i.e. cost analyses, cost-description studies, cost-outcome descriptions); and studies reporting more limited information, such as estimates of resource use or costs associated with service use are eligible for review.

\section{Types of participants}

- Adult ( $\geq 18$ years) patients admitted to an acute hospital for $>24$ hours and those in receipt of inpatient specialist palliative care while an inpatient in an acute hospital

- These patients will be diagnosed with advanced, lifelimiting or life-threatening illness (malignant or non-malignant), which is likely to compromise the patient's quality of life in some way

- Advanced illness occurs when one or more conditions become serious enough that general health and functioning decline, and treatments begin to lose their impact. This is a process that continues to the end of life (Coalition to Transform Advanced Care 2013)

- Diseases and health-related conditions included (with the corresponding International Classification of Diseases (ICD10)) are malignant neoplasms (ICD-10 codes: C00-C97) and non-malignant and other health-related conditions, specifically: heart disease, including cerebrovascular disease (ICD-10 codes: I00-I52, I60-69), renal disease (ICD-10 codes: N17, N18, N28, I12, I13), liver disease (ICD-10 codes: K70-K77), respiratory disease (ICD-10 codes: J06-J18, J20-22, J40-47, J96), neurodegenerative disease (Huntington's disease (ICD-10 code: G10), Parkinson's disease (ICD-10 code: G20), multiple sclerosis (ICD-10 code: G35), motor neuron disease (ICD-10 code: G12.2)), multi-system degeneration (ICD-10 code: G90.3), progressive supranuclear ophthalmoplegia (ICD-10 code: G23.1), Alzheimer's dementia and senility (ICD-10 codes: F01, F03, G20, R54), and/or HIV/AIDS (ICD-10 codes: B20-B24))

- Unpaid caregivers who have received a pre-bereavement intervention from one or more specialist palliative care staff in order to manage or alleviate bereavement-related problems prior to the death of the inpatient

- Unpaid caregivers are likely to be family, friends or significant others associated with the patient (Payne 2010a; Payne 2010b)

\section{Types of interventions}

Inpatient specialist palliative care varies between settings and countries. In order to allow for these differences, inpatient specialist palliative care will include care for patients with an advanced, lifelimiting or life-threatening illness that is likely to compromise the 
patient's quality of life in some way with or without pre-bereavement care for unpaid caregivers (provided while the patient is alive and in hospital to either the unpaid caregiver alone or together with the patient) (Higginson 2003). The intervention must be aiming to address the primary outcome of this review and/or a secondary outcome. It must also be delivered by a specialist palliative care team or by a "specialist palliative care", "palliative care" (but not a generalist palliative care member, as defined in Shipman 2008) or "hospice" staff member.

Comparisons will be made, where possible, with usual care. Usual care is defined as inpatient hospital care without any specialist palliative care input (for example, oncological care only), community care (for example, primary or specialist care provided in the patient's place of residence) or hospice care provided outside of the hospital setting. When usual care is compared with specialist palliative care (plus or minus usual care), we will extract descriptive data on what is involved in each intervention. Detailing these items will help address different implications regarding associated cost-effectiveness and costs in studies with various study designs and diverse specialist palliative care and usual care interventions. Similarly to a previous Cochrane systematic review that have examined palliative care (Gomes 2013), we will exclude trials evaluating inpatient specialist palliative care practitioners' provision of only a biomedical component of palliative care (for example, oxygen therapy) as this does not encompass the holistic nature of palliative care assessment or treatment. Focusing solely on a biomedical component may also counteract against the "protective" nature of inpatient specialist palliative care regarding unnecessary or aggressive medical treatment. In addition, in order to limit the size of this review and heterogeneity, specialist palliative care delivered to patients by outreach hospital services or within hospital outpatient services will not be included in the review. Specialist palliative care provided to unpaid caregivers in hospital settings and/or hospital outpatients will be included. This is because unpaid caregivers are likely to be seen as outpatients or in treatment rooms by hospital staff in the hospital to address pre-bereavement outcomes. Previous Cochrane reviews examining the effectiveness and cost-effectiveness of palliative care have been limited by the heterogeneity of both palliative care interventions and "usual care" (Gomes 2013). Limiting our review in this way will help limit heterogeneity.

\section{Types of outcome measures}

The primary and secondary outcomes for this review are developed from previous reviews regarding the effectiveness of palliative care (Gomes 2013; Gysels 2004; Higginson 2003; Higginson 2010). They reflect the multi-component nature of palliative care and the provision of both direct (e.g. face-to-face delivery of patient care) and indirect patient care (e.g. concerning practitioners' prescribing rationale), and care for unpaid caregivers while the inpatient is still alive. We have chosen to measure pain as our primary outcome rather than quality of life. Research has shown that conducting meta-analysis on data from instruments that do not measure the same underlying constructs or ones that differ substantially due to responsiveness (as is possible for patient-reported quality of life instruments) may be problematic, leading to between-study heterogeneity and biased meta-analysis (Puhan 2006). Pain control is a top priority for many potential palliative care patients and their unpaid caregivers in many countries (Bausewein 2013), and can be assessed by either the patient or by a proxy i.e. a healthcare clinician or an unpaid caregiver. The use of pain as the primary outcome incorporates a patient-level clinical outcome as central to the review.

\section{Primary outcomes}

- Pain, measured using validated assessment scales e.g. pain item of the Palliative care Outcome Scale

\section{Secondary outcomes}

- Patient other symptoms, specifically physical, psychological (for example, anxiety and/or depression or distress), social and/or spiritual domains, either patient or proxy-reported

- Quality of life

- Satisfaction with care

- Patient mortality/survival

- Unpaid caregiver symptom control, specifically physical, psychological (for example, anxiety and/or depression), social or spiritual domains, either unpaid caregiver or proxy-reported

- Unpaid caregiver burden, including emotional strain, burden, distress, mastery or positive aspects of caregiving

- Unpaid caregiver pre- and post-bereavement outcomes

- Cost outcomes:

- Inpatient hospital costs, including inpatient length of stay, consultations with healthcare professionals, investigations, treatments, equipment and medication prescribed by care provision (for example, usual care, specialist palliative care, usual care plus specialist palliative care)

- Unpaid caregiver costs from a societal perspective whereever possible (costs of caregivers' time off work, patient and caregivers' out-of-pocket expenses e.g. travel and child care costs, and any lost-opportunity costs);

- Measures of cost-effectiveness

- Economic evaluation outcome measures incorporating incremental cost effectiveness ratios using service cost data and condition specific outcome measures or quality-adjusted life years (QALYS) or an equivalent

- Adverse effects

- Increased clinical depression, increased psycho-socialemotional distress, and early and/or increased mortality

The effectiveness and cost-effectiveness of inpatient specialist palliative care in acute hospitals for adults with advanced illness and their 


\section{Search methods for identification of studies}

We will identify studies through e lectronic searches, handsearching, electronic citation tracking, personal contact and searching of grey literature. We will not place restrictions o $\mathrm{n}$ language ; $\mathrm{n}$ on-English papers will be assessed with the assistance of a native speaker, wherever possible. Where non-English studies are located and not able to be included in the review (due to a lack of resources to enable data extraction, for example), we will report accordingly to ensure transparency.

\section{Electronic searches}

We will identify studies by searching the databases listed below, using a combination of key terms and $\mathrm{MeSH}$ terms:

- Cochrane Library (Cochrane Central Register of Controlled Trials (CENTRAL), Cochrane Database of Systematic Reviews (CDSR), Database of Abstracts of Reviews of Effects (DARE), Health Technology Assessment (HTA)) (current issue);

- MEDLINE \& MEDLINE-in-Process (1947 to present);

- EMBASE (1974 to present);

- CINAHL (1981 to present);

- PsycINFO (1806 to present);

- CareSearch, Australian Government's Department of Health and Ageing (http://www.caresearch.com.au/) (from inception to present).

We will also search the following h ealth economic databases to identify further studies:

- National Health Service Economic Evaluation Database (NHS EED) (current issue);

- Health Economics Evaluation Database (HEED) (current issue);

- European Network of Health Economics Evaluation

Databases (EURONHEED) (1980 to present).

We will modify the MEDLINE search strategy for use in other databases (Appendix 1).

\section{Searching other resources}

\section{Handsearching}

We will screen the reference lists of all included studies and relevant reviews for additional studies.

\section{Electronic citation tracking}

We will use the "Citation tacking" option in MEDLINE for lateral searching on the included studies, as recommended for palliative care reviews (Payne 2010a).

\section{Personal contact}

When indicated to support data analysis, we will attempt to contact key investigators identified from the included studies for unpublished data or knowledge of grey literature. The collective knowledge of the Cochrane Pain, Palliative and Supportive Care Group editorial team will also be used to identify potential investigators and their studies to approach regarding unpublished data and their knowledge of grey literature.

\section{Data collection and analysis}

\section{Selection of studies}

Two authors (BD, MS) will independently screen all titles and abstracts identified in our electronic searches. If, after reading the abstract, doubt persists regarding the eligibility of the study, we will retrieve the full-text articles for further assessment and again these full-text articles will be assessed by the two authors independently. A third author (CE) will adjudicate any discrepancies between the two authors' assessment of eligibility. Disagreements will be resolved by discussion and consensus. We plan to illustrate our study selection process using a Preferred Reporting Items for Systematic Reviews and Meta-Analyses (PRISMA) flow diagram (Liberati 2009), as recommended in the Cochrane Handbook for Systematic Reviews of Interventions (Higgins 2011).

\section{Data extraction and management}

Two authors (BD, MS) will independently extract and enter data from all included studies using a data extraction form developed for the review (Appendix 2). Disagreements will be resolved by discussion and consensus with a third author (CE). The data extraction form has been used previously for a review on the effectiveness of home palliative care (Gomes 2013). The form has been adapted for this review regarding inpatient specialist palliative care. Drawing on an existing data extraction form enables future work comparing the effectiveness and cost-effectiveness of specialist palliative care across care settings.

\section{Assessment of risk of bias in included studies}

Two authors (BD, MS) will independently assess the quality of all selected RCTs using the Cochrane Effective Practice and Organisation of Care (EPOC) criteria for effectiveness studies (EPOC 2015). For non-randomised studies, we will use the 'Cochrane Risk Of Bias Assessment Tool for Non-Randomized Studies of Interventions' (Sterne 2014). For full economic evaluations, we will use a 35-item checklist employed by BMJ for authors and peer reviewers of economic submissions; a shorter version of this checklist will be used for partial economic evaluation (Drummond 1996). In order to identify low quality evaluation, each item of the 
checklist will be equally weighted and scored either 1 (addressed), 0.5 (unclear) and 0 (not addressed) (Gonzalez-Perez 2002) and in order to allow for appraisal of each component of selected studies, we will complete an average score for each section (study design, data collection, analysis and interpretation of results). This is to avoid an overall average score, which may mask specific areas of weakness. If useful, we will also calculate an overall average score to provide some overall indication of quality. As used previously in palliative care systematic reviews (Gomes 2013), an average score under 0.6 will be considered as low quality (Gonzalez-Perez 2002). We will assess the quality of relevant economic modelling studies using the Philips Checklist (Philips 2004).

We will assess risk of bias in all other studies using the Cochrane Collaboration's tool for assessing risk of bias (Higgins 2011), which involves assessment of individual domains, such as allocation concealment and blinding. Blinding may not always be possible for many palliative care studies. In addition, as with any research project, attempts at blinding may be unsuccessful once implementation occurs. We will examine blinding in each study, including whether this occurred and whether it was compromised during study implementation. These factors will be considered when interpreting the results of the review. Similarly, we will report selection, performance, detection, attrition, reporting and other sources of bias (where relevant). A summary of bias in relation to each important outcome and also across studies will be provided as either low risk, unclear risk or high risk. We will prepare 'Risk of bias' tables using the Cochrane Collaboration's statistical software, Review Manager 2014.

Cochrane guidance provides scope for assessing the risk of bias based on the likelihood that the outcome will be influenced by lack of blinding. The guidance suggests that a common assessment of risk be completed for all subjective outcomes (e.g. distress) as compared to objective outcomes (e.g. mortality) (Higgins 2011). Accordingly, we will group all "subjective" primary and secondary outcomes as being at high-risk of bias if blinding is unsuccessful. However, the mortality outcome is unlikely to be influenced by lack of blinding. This will therefore be treated as a "low risk of bias" even if blinding is unsuccessful.

\section{Measures of treatment effect}

If appropriate, we will undertake meta-analysis of the primary and secondary outcomes using Review Manager 2014. We will evaluate the direction and size of the effect as well as looking at the consistency of the effect across the selected studies. We will appraise the strength of the evidence using the grading system recommended by the Cochrane Collaborative (Van Tulder 2003). To measure treatment effect, we will calculate a summary statistic for each study followed by an overall average treatment effect. We will use o dds ratios (ORs) with 95\% confidence intervals (CIs) for each study to determine whether pain was reduced or not. Our primary outcome will be treated as a binary outcome. This will aid interpretation of the findings for clinicians and address the heterogeneity of pain data. Pain outcome data in eligible studies is likely to be presented as binary or ordinal data. This will involve transforming data by aggregating adjacent categories. Decisions related to dichotomising data will be informed primarily by clinical considerations with reference to the study population. Even though ORs will be used to detect treatment effect, we will present findings as risk ratios (RRs) (or relative risk reduction) in order to aid the use and interpretation of the findings by end users.

We will use either a fixed-effect or a random-effects model for meta-analysis. It is likely that a random-effects model will be used as the true effect size may not be due to chance alone. Eligible studies will most probably have been conducted with different populations, countries and years, for example. It is therefore likely that we may incorporate the assumption of heterogeneity in our review. However, should one true underlying fixed effect size be detected, we will use a fixed-effect model.

For secondary outcomes, we will either calculate standardi sed mean differences (SMDs) with 95\% cCIs in both intervention(s) and comparator(s) in order to show the intervention effect involving continuous data. For measures in the form of binary data, we will calculate ORs with 95\% CIs. Ordinal data may be transformed into dichotomous data by aggregating adjacent categories together (informed by clinical judgement). If the same continuous outcome measure is used in all studies and measured in the same way, then the results will be averaged and we will calculate a mean difference (MD). If the outcome measures are the same, but they are measured differently in each study, we will calculate the SMD. A SMD of less than or equal to 0.2 will constitute a small effect, 0.2 to 0.5 will constitute a moderate effect and $\geq 0.8$ will constitute a large effect. Statistical significance will be assumed using a $P$ value of $<0.05$.

\section{Measures of resource use and cost}

We will present characteristics of the included health economics studies, such as year of study; details of interventions and comparators; study design; data sources; jurisdiction and setting; analytic perspective and time horizon, in the 'Characteristics of included studies' table as recommended in the Cochrane Handbook for Systematic Reviews of Interventions (Higgins 2011). We will provide a dditional tables, which summarise checklists completed to inform assessments of the methodological quality of included health economics studies. We will present $\mathrm{p}$ oint estimates of measures of items of resource use and cost with associated measures of uncertainty for both the target intervention and each of its comparators, as well as point estimates of incremental costs and/or costeffectiveness, again with associated measures of uncertainty. We will convert c osts to US dollars (USD) or Great British Pounds (GBP) (current year) based on Purchasing Power Parities (PPP) and gross domestic product (GDP) deflators. 


\section{Cost-effectiveness}

We will identify and report incremental cost per QALY (or equivalent) and cost-benefit ratios where relevant. We will conduct $f$ urther cost-effectiveness analysi s depending on the level of data retrieved.

\section{Unit of analysis issues}

Issues in the analysis of studies with particular characteristics, for example cross-over trials and cluster randomised trials, will be addressed once, and if, such studies are identified. We will report intra-cluster correlations for cluster trials and adjustments will be completed where necessary. We intend to use the intra-class correlation coefficient (ICC) supplied in eligible studies to adjust for meta-analysis. If not supplied in the article, we will seek this information from the study authors. If still unavailable, we will estimate an intra-class correlation to allow for meta-analysis.

\section{Dealing with missing data}

When data is missing from a study, we will contact the original investigator for clarification and additional information where possible. Any strategy used for imputing missing data will be described, as well as justifying the choice of the strategy used. We are also expecting to find studies with missing intervention data (number of staff involved, skills and so on). The potential impact of this missing data on the findings of the review will be examined in the discussion section. We will seek clarity from authors regarding study population and interventions where required, especially to aid examination of the components of the intervention.

\section{Assessment of heterogeneity}

We will examine and assess h eterogeneity through the following three measures:

1. inspecting the studies to examine for plausible areas of heterogeneity based on clinical factors that may influence findings of our meta-analysis;

2. inspecting the forest plots;

3. using the the $\mathrm{I}^{2}$ statistics to examine the extent and impact of heterogeneity between included studies.

We will explore reasons for heterogeneity $\mathrm{n}$ sensitivity analysis should high heterogeneity be identified ( $\mathrm{I}^{2} \geq 75 \%$ ) (Higgins 2011).

\section{Assessment of reporting biases}

In order to detect and manage reporting bias, we will take steps to attend to:

- multiple (publication) bias through contacting authors to ascertain whether duplication has occurred;
- location bias by searching relevant national and international trial registries for all relevant studies included (e.g. CENTRAL);

- language bias by including studies published in languages other than English, where possible, and if their inclusion is not feasible then we will report on these studies to identify that their data was not included in the review; and

- outcome reporting will be addressed through comparing the findings in eligible studies with published protocols where available.

In addition, if there are more than 10 included studies in our metaanalysis, we will use funnel plots and visually inspect them for a/ symmetry as means of determining the effects of any eligible small study. We will also conduct relevant tests for asymmetry influenced by data type (e.g. continuous or dichotomous), to assist with examining publication bias and to overcome any reliance on visual inspection (Lau 2006). Should small-study effects be identified, we will conduct sensitivity analysis to examine different assumptions and their impact on the review findings. We will determine fixed-effect and random-effects estimates of the intervention effect if it becomes evident that there is between-study heterogeneity. When asymmetry is observed, we will consider publication bias as one (of several) plausible explanations (Sterne 2001).

As the potential for bias is greater in a non-randomised study than in a well-conducted randomised trial (Kato 1999; Strobe 2001; Wright 2006), we will pay particular attention to selection bias and reporting bias for non-randomised studies. We will critically appraise all studies and assess their risk of bias (Higginson 2008b). Non-randomised studies are generally assessed as low in quality, but can be appraised higher if indicated by a large magnitude of effect or lack of concern about confounding (Higginson 2008b). We will assess risk of bias in each included study and document our findings for judgement record (e.g. study design characteristics) (Higginson 2008b).

\section{Data synthesis}

Should the eligible studies not be sufficiently homogenous to permit meta-analysis, we will extract quantitative data (means, standard deviations, frequencies and proportions, test coefficients, $95 \%$ confidence intervals and effects sizes, where available) and techniques used in narrative synthesis will be employed to analyse the data, including:

- abulation, which will involve inserting the main elements of extracted data into a table format;

- textual descriptions, which will involve collating a summary description of each included study;

- clustering of group textual descriptions according to attributes; and

- vote counting to determine how often certain attributes were reported (Rodgers 2009).

The effectiveness and cost-effectiveness of inpatient specialist palliative care in acute hospitals for adults with advanced illness and their 
Where possible, we will include qualitative data from nested or embedded qualitative studies reported on within eligible studies and analyse them through narrative synthesis methods.

\section{Subgroup analysis and investigation of heterogeneity}

As part of our primary objective, we will be identifying the effective components and determining the comparative effectiveness of inpatient specialist palliative care in acute hospitals for adults with advanced illnes $s$ and their caregivers. We will compare the resources and costs associated with these services and determine their cost-effectiveness; compare the effectiveness by disease type (e.g. malignant and non-malignant groups) inpatient settings and country; examine other sources of heterogeneity, including interventions offering only single or few components of palliative care, and the applicability of meta-analysis.

We will perform subgroup analysis using the following components known to influence the effectiveness of inpatient specialist care and in relation to particular patient groups.

1. Patient characteristic of disease type, including malignant and non-malignant disease to improve the evidence base for different types of palliative care populations (Higginson 2010). Those with malignant disease will be those diagnosed with malignant neoplasms (ICD-10 codes: C00-C97). Those with non-malignant and other health-related conditions, will include those diagnosed with: heart disease, including cerebrovascular disease (ICD-10 codes: I00-I52, I60-69), renal disease (ICD-10 codes: N17, N18, N28, I12, I13), liver disease (ICD-10 codes: K70-K77), respiratory disease (ICD-10 codes: J06-J18, J20-22, J40-47, J96), neurodegenerative disease (Huntington's disease (ICD-10 code: G10), Parkinson's disease (ICD-10 code: G20), multiple sclerosis (ICD-10 code: G35), motor neuron disease (ICD-10 code: G12.2)), multi-system degeneration (ICD-10 code: G90.3), progressive supranuclear ophthlmolegia (ICD-10 code: G23.1), Alzheimer's dementia and senility (ICD-10 codes: F01, F03, G20, R54), and / or HIV/AIDS (ICD-10 codes: B20B24).

2. Frailty associated with advanced age due to how valuable these findings will be to society and future commissioning of services.

3. Inpatient specialist palliative care team composition (for example, physician-led as compared to nurse-led palliative care services) and organisation (for example, 24-hour access versus temporally restricted access) to examine the effectiveness of different models of service provision and to inform service delivery and configuration. This subgroup analysis will aid the identification of key components of inpatient specialist palliative care models (Higginson 2010).

4. Country of origin will also be explored due to differences in care structures and the availability of inpatient specialist palliative care, and any associated impact of this on effectiveness and cost-effectiveness.

\section{Sensitivity analysis}

We plan to conduct sensitivity analyses due to heterogeneity related to clinical (e.g. intervention type, patient population) and statistical reasons inherent within eligible studies. The $\mathrm{I}^{2}$ statistic will help us examine the extent and impact of heterogeneity between included studies. We will explore reasons for heterogeneity using sensitivity analysis when high heterogeneity $\left(\mathrm{I}^{2} \geq 75 \%\right)$ is evident (Higgins 2011).

\section{ACKNOWLEDGEMENTS}

We acknowledge Hamid Benalia, Emily West, Sue Hall, Barbara Gomes and Nancy Preston who contributed to earlier drafts of this protocol.

BuildCARE members: Emma Bennett, Francesca Cooper, Barbara Daveson, Susanne de Wolf-Linder, Mendwas Dzingina, Clare Ellis-Smith, Catherine Evans, Taja Ferguson, Lesley Henson, Irene Higginson, Bridget Johnston, Pauline Kane, Peter Lawlor, Paul McCrone, Regina McQuillan, Diane Meier, Sean Morrison, Fliss Murtagh, Charles Normand, Steve Pantilat, Ana Reison, Karen Ryan, Lucy Selman, Melinda Smith, Katy Tobin, Rowena Vohora, Gao Wei.

Cochrane Review Group funding acknowledgement: The National Institute for Health Research (NIHR) is the largest single funder of the Cochrane PaPaS Group. Disclaimer: The views and opinions expressed in this review are those of the authors and do not necessarily reflect those of the NIHR, National Health Service (NHS) or the Department of Health. 


\section{R E F E R E N C E S}

\section{Additional references}

\section{AIHW 2014}

Australian Institute of Health and Welfare (AIHW) 2014.

Health expenditure Australia 2011-12: analysis by sector. Health and welfare expenditure series no. 51. Cat. no. HWE 60. Canberra: AIHW. Available from http:// www.aihw.gov.au/publication-detail/?id=60129550226.

Allen 2008

Allen RS, Hilgeman MM, Ege MA, Shuster Jr. JL, Burgio LD. Legacy activities as interventions approaching the end of life. Journal of Palliative Medicine 2008;11(7):1029-38.

\section{Bausewein 2013}

Bausewein C, Calanzani N, Daveson BA, Simon ST, Ferreira PL, Higginson IJ, et al. 'Burden to others' as a public concern in advanced cancer: a comparative survey in seven European countries. BMC Cancer 2013;13(1):105.

\section{Bodenbach 2005}

Bodenbach M. Developing a bereavement services component for an urban teaching hospital's new palliative care program: a four-target survey approach. Journal of Palliative Medicine 2005;8(4):713-5.

Center to Advance Palliative Care 2014

Center to Advance Palliative Care. Growth of palliative care in U.S. hospitals 2014 snapshot. Available from https://www.capc.org/media/filer_public/6d/db/6ddbbaa10c03-4e34-9186-696bbc158950/capc_growth_snapshot_ 2014.pdf 2014.

Coalition to Transform Advanced Care 2013 Coalition to Transform Advanced Care (CTAC). Policy framework. Available from http:// advancedcarecoalition.org/wp-content/uploads/2011/10/ Policy-Framework.pdf 2013.

\section{Daveson 2014a}

Daveson BA, Harding R, Shipman C, Mason BL, Epiphaniou E, Higginson IJ, et al. The real-world problem of care coordination: a longitudinal qualitative study with patients living with advanced progressive illness and their unpaid caregivers. PLoS ONE 2014;99(5):e95523. [DOI: 10.1371/journal.pone.0095523]

\section{Daveson 2014b}

Daveson BA, Alonso JP, Calanzani N, Ramsenthaler C, Gysels M, Antunes B, et al. on behalf of PRISMA. Learning from the public: citizens describe the need to improve endof-life care in Europe. European Journal of Public Health 2014;24(3):521-7.

DoH 2008

Department of Health. End of Life Care Strategy: Promoting high quality care for adults at the end of their life. 16 July 2008. Available from https://www.gov.uk/ government/publications/end-of-life-care-strategypromoting-high-quality-care-for-adults-at-the-end-of-theirlife.

\section{Drummond 1996}

Drummond M, Jefferson T. Guidelines for authors and peer reviewers of economic submissions to the BMJ. BMJ 1996; 313(7052):275-83.

\section{EPOC 2015}

Effective Practice and Organisation of Care Group (EPOC). Suggested risk of bias criteria for EPOC reviews. EPOC Resources for review authors. Oslo: Norwegian Knowledge Centre for the Health Services; 2015. Available at: http:// epoc.cochrane.org/epoc-specific-resources-review-authors.

Evans 2014

Evans CJ, Ho Y, Daveson BA, Hall S, Higginson IJ, Gao W, GUIDE_Care project. Place and cause of death in centenarians: a population based observational study in England, 2001 to 2010. PLoS Medicine 2014;11(6): e1001653. [DOI: 10.1371/journal.pmed.1001653]

Field 2004

Field D, Reid D, Payne S, Relf M. Survey of UK hospice and specialist palliative care adult bereavement services. International Journal of Palliative Nursing 2004;10(12): 569-76.

Gomes 2013

Gomes B, Calanzani N, Curiale V, McCrone P, Higginson IJ. Effectiveness and cost-effectiveness of home palliative care services for adults with advanced illness and their caregivers. Cochrane Database of Systematic Reviews 2013, Issue 6. [DOI: 10.1002/14651858.CD007760]

Gonzalez-Perez 2002

Gonzalez-Perez JG. Developing a scoring system to quality assess economic evaluations. European Journal of Health Economics 2002;3(2):131-6.

Gruneir 2007

Gruneir A, Mor V, Weitzen S, Truchil R, Teno J, Roy J. Where people die: a multilevel approach to understanding influences on site of death in America. Medical Care Research and Review 2007;64(4):351-78.

Gysels 2004

Gysels M, Richardson A, Higginson IJ. Communication training for health professionals who care for patients with cancer: a systematic review of their effectiveness. Supportive Care in Cancer 2004;12(10):692-700.

Harding 2012

Harding R, Epiphaniou E, Chidgey-Clark J. Needs, experiences, and preferences of sexual minorities for end-oflife care and palliative care: a systematic review. Journal of Palliative Medicine 2012;15(5):602-11.

Harris 2013

Harris I, Murray SA. Can palliative care reduce futile treatment? A systematic review. BMJ Supportive \& Palliative Care 2013;3(4):389-98.

\section{Hatziandreu 2008}

Hatziandreu E, Archontakis F, Daly A. The potential cost savings of greater use of home- and hospice- based end

The effectiveness and cost-effectiveness of inpatient specialist palliative care in acute hospitals for adults with advanced illness and their 
of life care in England. Prepared for the National Audit Office. Published 2008 by the RAND Corporation. http:// www.rand.org/pubs/technical_reports/TR642.html.

Higgins 2011

Higgins JPT, Green S (editors). Cochrane Handbook for Systematic Reviews of Interventions Version 5.1.0 [updated March 2011]. The Cochrane Collaboration, 2011. Available from www.cochrane-handbook.org.

\section{Higginson 2003}

Higginson IJ, Finlay IG, Goodwin DM, Hood K, Edwards AGK, Cook A, et al. Is there evidence that palliative care teams alter end-of-life experiences of patients and their caregivers?. Journal of Pain and Symptom Management 2003; 25(2):150-68.

Higginson 2008b

Higginson IJ, Hart S, Burman R, Silber, E, Saleem T, Edmonds P. Randomised controlled trial of a new palliative care service: compliance, recruitment and completeness of follow-up. BMC Palliative Care 2008;7:7. [DOI: 10.1186/ 1472-684X-7-7]

Higginson 2010

Higginson IJ, Evans C. What is the evidence that palliative care teams improve outcomes for cancer patients and their families?. Cancer Journal 2010;16(5):423-35.

Higginson 2011

Higginson IJ, Costantini M, Silber E, Burman R, Edmonds P. Evaluation of a new model of short-term palliative care for people severely affected with multiple sclerosis: a randomised fast-track trial to test timing of referral and how long the effect is maintained. Postgraduate Medical Journal 2011;87(1033):769-75.

Ho 2011

Ho TH, Barbera L, Sakin R, Lu H, Neville BA, Earle CC. Trends in the aggressiveness of end-of-life cancer care in the universal health care system of Ontario, Canada. Journal of Clinical Oncology 2011;29(12):1587-91.

\section{Hudson 2005}

Hudson PL, Aranda S, Hayman-White K. A psychoeducational intervention for family caregivers of patients receiving palliative care: a randomized controlled trial. Journal of Pain and Symptom Management 2005;30(4): $329-41$.

Kamal 2013

Kamal AH, Currow DC, Ritchie CS, Bull J, Abernathy AP. Community-based palliative care: the natural evolution for palliative care delivery in the U.S.. Journal of Pain and Symptom Managment 2013;46(2):254-64.

Kashihara 2012

Kashihara D, Carper K. National Health Care Expenses in the U.S. Civilian Noninstitutionalized Population, 2009. Statistical Brief \#355. January 2012. Agency for Healthcare Research and Quality, Rockville, MD. Available from http: //www.meps.ahrq.gov/mepsweb/data_files/publications/ st355/stat355.shtml.
Kato 1999

Kato PM, Mann T. A synthesis of psychological intervention for the bereaved. Clinical Psychology Review 1999;19(3): 275-96.

\section{Lafond 2014}

Lafond S, Arora S, Charlesworth A, McKeon A. Into the red? The state of the NHS' finances. An analysis of NHS expenditure between 2010 and 2014. Nuffield Trust research report. July 2014. Available from http:// www.nuffieldtrust.org.uk/sites/files/nuffield/publication/ into_the_red.pdf.

Lau 2006

Lau J, Ioannidis JP, Terrin N, Schmid CH, Olkin I. The case of the misleading funnel plot. BMJ 2006;333(7568): 597-600.

\section{Liberati 2009}

Liberati A, Altman DG, Tetzlaff J, Mulrow C, Gotzsche PC, Ioannidis JPA, et al. The PRISMA statement for reporting systematic reviews and meta-analyses of studies that evaluate health care interventions: explanation and elaboration. Annals of Internal Medicine 2009;151(4):W65-94.

\section{Lichtenthal 2011}

Lichtenthal WG, Nilsson M, Kissane DW, Breitbart W, Kacel E, Jones EC, et al. Underutilization of mental health services among bereaved caregivers with prolonged grief disorder. Psychiatric Services 2011;62(10):1225-9.

\section{McMillan 2006}

McMillan SC, Small BJ, Weitzner M, Schonwetter R, Tittle $\mathrm{M}$, Moody L, et al. Impact of coping skills intervention with family caregivers of hospice patients with cancer: a randomized clinical trial. Cancer 2006;106(1):214-22.

\section{Meier 2011}

Meier DE. Increased access to palliative care and hospice services: opportunities to improve value in health care. Millbank Quarterly 2011;89(3):343-80.

Morrison 2008

Morrison RS, Penrod JD, Cassel JB, Caust-Ellenbogen M, Litke A, Spragens L, et al. Cost savings associated with US hospital palliative care consultation programs. Archives of Internal Medicine 2008;168(16):1783-90.

\section{Murray 2005}

Murray SA, Kendall M, Boyd K, Sheikh A. Illness trajectories and palliative care. BMJ 2005;330:1007.

\section{Murray 2012}

Murray CJL, Vos T, Lozano R, Naghavi M, Flaxman AD, Michaud C, et al. Disability-adjusted life years (DALYs) for 291 diseases and injuries in 21 regions, 1990-2010: a systematic analysis for the Global Burden of Disease Study 2010. Lancet 2012;380(9859):2197-223.

\section{Murtagh 2014}

Murtagh FE, Bausewein C, Verne J, Groeneveld EI, Kaloki YE, Higginson IJ. How many people need palliative care? A study developing and comparing methods for populationbased estimates. Palliative Medicine 2014;28(1):49-58.

The effectiveness and cost-effectiveness of inpatient specialist palliative care in acute hospitals for adults with advanced illness and their 


\section{NCP 2013}

National Consensus Project for Quality Palliative Care. Clinical Practice Guidelines for Quality Palliative Care. 3rd ed. Pittsburgh, PA: National Consensus Project for Quality Palliative Care; 2013. Available at: http: //www.nationalconsensusproject.org/NCP_Clinical_ Practice_Guidelines_3rd_Edition.pdf.

\section{NCPC 2012}

National Council for Palliative Care. Commissioning Guidance for Specialist Palliative Care: Helping to deliver commissioning objectives. Guidance document published collaboratively with the Association for Palliative Medicine of Great Britain and Ireland, Consultant Nurse in Palliative Care Reference Group, Marie Curie Cancer Care,National Council for Palliative Care, and Palliative Care Section of the Royal Society of Medicine, London, UK. December 2012. Available from http://www.ncpc.org.uk/publication/ commissioning-guidance-specialist-palliative-care-helpingdeliver-commissioning-objectiv.

\section{NIH 2004}

National Institute of Health. NIH State-of-the-Science Conference Statement on improving end-of-life care. NIH Consensus and State-of-the-Science Statements 2004;21(3): $1-26$.

Obermeyer 2014

Obermeyer Z, Makar M, Abujaber S, Dominici F, Block $S$, Cutler DM. Association between the Medicare hospice benefit and health care utilization and costs for patients with poor-prognosis cancer. JAMA 2014;312(18):1888-96.

Palliative Care Australia 2005

Palliative Care Australia. A Guide to Palliative Care Service Development: A population based approach. February 2005. Available from http://www.palliativecare.org.au/Portals/46/ A\%20guide $\% 20$ to $\% 20$ palliative $\% 20$ care $\% 20$ service $\% 20$ development $\% 20$ $\% 20 \mathrm{a} \% 20$ population $\% 20$ based\%20approach.pdf.

\section{Payne 2010a}

Payne S, Hudson P, Grande G, Oliviere D, Tishelman C, Pleschberger S, et al. EAPC Task Force on Family Carers White Paper on improving support for family carers in palliative care: part 1. European Journal of Palliative Care 2010;17(5):238-45.

Payne 2010b

Payne S, Hudson P, Grande G, Oliviere O, Tishelman C, Pleschberger $S$, et al. EAPC Task Force on Family Carers White Paper on improving support for family carers in palliative care: part 2. European Journal of Palliative Care 2010;17(6):286-90.

Philips 2004

Philips Z, Ginnelly L, Sculpher M, Claxton K, Golder $S$, Riemsma R, et al. Review of guidelines for good practice in decision-analytic modelling in health technology assessment. Health Technology Assessment 2004;8:1-172.

Pinnock 2011

Pinnock H, Kendall M, Murray SA, Levack P, Porter $\mathrm{M}, \mathrm{MacNee} \mathrm{W}$, et al. Living and dying with severe chronic obstructive pulmonary disease: multi-perspective longitudinal qualitative study. BMJ 2011;342:d142.

Puhan 2006

Puhan MA, Soesilo I, Guyatt GH, Schunemann HJ. Combining scores from different patient reported outcome measures in meta-analyses: when is it justified?. Health and Quality of Life Outcomes 2006;4:94. [DOI: 10.1186/ 1477-7525-4-94]

\section{Quill 2013}

Quill TE, Abernathy AP. Generalist plus specialist palliative care - creating a more sustainable model 2013. New England Journal of Medicine 2013;268(13):1173-5.

Reid 2006

Reid D, Field D, Payne S, Relf M. Adult bereavement in five English hospices: types of support. International Journal of Palliative Nursing 2006;12(9):430-7.

\section{Review Manager 2014}

The Nordic Cochrane Centre, The Cochrane Collaboration. Review Manager (RevMan). 5.3. Copenhagen: The Nordic Cochrane Centre, The Cochrane Collaboration, 2014.

\section{Rinck 1997}

Rinck GC, van den Bos GA, Kleijnen J, de Haes HJ, Schade E, Veenhof $\mathrm{CH}$. Methodologic issues in effectiveness research on palliative cancer care: a systematic review. Journal of Clinical Oncology 1997;15(4):1697-707.

\section{Rodgers 2009}

Rodgers M, Sowden A, Petticrew M, Arai L, Roberts H, Britten N, et al. Testing methodological guidance on the conduct of narrative synthesis in systematic reviews. Evaluation 2009;15(1):47-71.

\section{Shipman 2008}

Shipman C, Gysels M, White P, Worth A, Murray SA, Barclay $S$, et al. Improving generalist end of life care: national consultation with practitioners, commissioners, academics, and service user groups. BMJ 2008;337:a1720.

\section{Smith 2012}

Smith SM, Soubhi H, Fortin M, Hudon C, O'Dowd T. Managing patients with multimorbidity: systematic review of interventions in primary care and community settings. BMJ 2012;345:e5205.

Sterne 2001

Sterne JAC, Egger M, Smith GD. Investigating and dealing with publication and other biases in meta-analysis. $B M J$ 2001;323:101-5.

Sterne 2014

Sterne JAC, Higgins JPT, Reeves BC on behalf of the development group for ACROBAT-NRSI. A Cochrane Risk Of Bias Assessment Tool: for Non-Randomized Studies of Interventions (ACROBAT-NRSI), Version 1.0.0, 24 September 2014. Available from http://www.riskofbias.info (accessed 20 November 2014).

Strobe 2001

Strobe MS, Hansson RO, Stroebe W, Schut H. Introduction: concepts and issues in contemporary research

The effectiveness and cost-effectiveness of inpatient specialist palliative care in acute hospitals for adults with advanced illness and their 
on bereavement. In: Stroebe MS, Hansson RO, Stroebe W, Schut H editor(s). Handbook of Bereavement Research: Consequences, Coping and Care. 1st Edition. Washington DC: American Psychological Association, 2001:3-22.

\section{Sullivan 2011}

Sullivan R, Peppercorn J, Sikora K, Zalcberg J, Meropol NJ, Amir E, et al. Delivering affordable cancer care in highincome countries. Lancet Oncology 2011;12(10):933-80.

\section{Temel 2010}

Temel JS, Greer JA, Muzikansky A, Gallagher ER, Admane S, Jackson VA, et al. Early palliative care for patients with metastatic non-small-cell lung cancer. New England Journal of Medicine 2010;363(8):733-42.

\section{The WHOQOL Group 1995}

The WHOQOL Group. The World Health Organization Quality of Life Assessment (WHOQOL): position paper from the World Health Organization. Social Science and Medicine 1995;41(10):1403-9.

Van Tulder 2003

Van Tulder M, Furlan A, Bombardier C, Bouter L. Updated method guidelines for systematic reviews in the Cochrane Collaboration Back Group. Spine 2003;28(12):1290-9.

\section{Walsh 2011}

Walsh J, Young JM, Harrison JD, Butow PN, Solomon MJ, Masya L, et al. What is important in cancer care coordination? A qualitative investigation. European Journal of Cancer Care 2011;20(2):220-7.

\section{WPCA/WHO 2014}

World Palliative Care Alliance, World Health Organization. Global Atlas of Palliative Care at the End of Life. January 2014. Available from http://www.who.int/cancer/ publications/palliative-care-atlas/en/.

\section{Wright 2006}

Wright DNM, Hopkinson JB, Corner JL, Foster CL. How to involve cancer patients at the end of life as co-researchers. Palliative Medicine 2006;20:821-7.

\section{Zhao 2010}

Zhao Y, Encinosa W. The Costs of End-of-Life Hospitalizations, 2007. November 2009, revised April 2010, Statistical Brief \#81. Healthcare Cost and Utilization Project (HCUP). Agency for Healthcare Research and Quality, Rockville, MD. Available from www.hcupus.ahrq.gov/reports/statbriefs/sb81.jsp.

\section{Zimmerman 2014}

Zimmerman C, Swami N, Kryzanowska M, Hannon B, Leighl N, Oza A, et al. Early palliative care for patients with advanced cancer: a cluster-randomised controlled trial. Lancet 2014;383(9930):17-23.

* Indicates the major publication for the study

\section{A P P E N D I C ES}

\section{Appendix I. MEDLINE search strategy}

1. exp palliative care/

2. exp terminal care/

3. exp terminally ill/

4. palliat*.mp.

5. (terminal* adj3 (care or caring)).mp.

6. ((advanced or end stage or terminal) adj3 (disease* or ill* or cancer* or malignan*)).mp.

7. (last year of life or LYOL or life's end or end of life).mp.

8. or/1-7

9. exp hospitals/

10. inpatients/

11. ((hospital* or inpatient*) adj2 (base* or care or center* or centre* or interven* or management or model* or nurs* or program* or service* or team* or therap* or treat*)).mp.

12. or/9-11

13. 8 and 12

14. randomized controlled trial.pt.

15. controlled clinical trial.pt.

16. randomized.ab.

17. placebo.ab.

18. drug therapy.fs.

The effectiveness and cost-effectiveness of inpatient specialist palliative care in acute hospitals for adults with advanced illness and their 
19. randomly.ab.

20. trial.ab.

21. groups.ab.

22. (random* or control* or intervention* or evaluat*).tw.

23. ((before and after) or case control* or cohort study or or quasi experiment* or time series).tw.

24. or/14-23

25. 13 and 24

26. exp budgets/ or exp "costs and cost analysis"/ or economics/ or exp economics, hospital/ or exp economics, medical/ or economics, nursing/ or exp "fees and charges"/ or exp resource allocation/ or value of life/

27. (cost* or economic*).ti. or (cost* adj2 (effective* or utilit* or benefit* or minimi*)).ab. or economic model*.tw. or (budget* or fee* or financ* or price* or pricing or resourc* allocat* or (value adj2 (monetary or money))).ti,ab.

28. or/26-27

29. 13 and 28

30. 25 or 29

31. (animals not (humans and animals)).sh.

32. not 31

33.32 and 30

\section{Appendix 2. Items to be included in the data extraction form}

\section{Study details}

- Publication details (author/s, year, journal)

- Country of origin

- Verification of the study eligibility

- Aim/hypothesis

- Type of hospital

\section{Study design and methods}

- Study design

- Type of intervention and control (if used)

- Inclusion/exclusion criteria

- Allocation sequence procedures

- Allocation concealment

- Type of blinding

- Details of blinding (including instances of blinding being compromised)

- Data collection period

- Baseline measurement/s

- Number of follow-ups

- Time that follow-ups occurred

- Sample size (number in each group)

- Sample size calculations

- Outcome measures used (differentiating primary and secondary)

- Recruitment rate

- Method of analysis

- Method of managing missing data

- Study participant characteristics for patient and/or unpaid caregiver (e.g., age, sex, race, sexual orientation, diagnosis)

- Selective reporting

The effectiveness and cost-effectiveness of inpatient specialist palliative care in acute hospitals for adults with advanced illness and their 


\section{Intervention(s) and comparator(s)}

- Setting of intervention

- Type of intervention

- Staff composition

- Staff training and experience

- Components of intervention

- Frequency of intervention

- Duration of intervention

\section{Primary outcome}

- Measurement and change in pain

\section{Secondary outcomes}

- Measurement and change in patient symptoms other than pain (e.g., quality of life)

- Measurement and change in unpaid caregiver symptoms

- Measurement and change in unpaid caregiver burden

- Patient and/or unpaid caregiver mortality

- Proportion of time that the patient spends admitted as an inpatient

\section{Costs (resource use)}

- Health care cost

- Cost data sources

- Cost analytical perspective

\section{Additional items}

- Adverse effects

- Number and reason for withdrawals

- Number of drop-outs

- Preferred place of death data

- Data related to availability of other services in the local area, in particular data related to palliative home care services

\section{CONTRIBUTIONSOFAUTHORS}

All review authors: contributed to the development of the idea and of the protocol.

BD, CE, MS: developed and wrote the protocol, developed the search strategies and the data extraction form, which built upon previous palliative care Cochrane reviews.

CT, MC, FM: discussed the protocol and contributed to its development along with the other members of the review team, as well as contributing to the development of the search strategy and the data extraction form. 


\section{DECLARATIONSOF INTEREST}

All members of this review team work to advance palliative care through robust science. It is likely that a study authored or co-authored by one or more than one of the review authors may be included in this review. There are no other declarations of interest to declare.

\section{SOURCES OF SUPPORT}

\section{Internal sources}

- Department of Palliative Care, Policy and Rehabilitation, Cicely Saunders Institute, King's College London, London, UK.

- Institute of Psychiatry, King's College London, London, UK.

\section{External sources}

- School of Nursing, Midwifery and Social Work, University of Manchester, Manchester, UK.

- Amsterdam Institute of Social Science Research, University of Amsterdam, Amsterdam, Netherlands.

- Regional Palliative Care Network, IRCCS AOU San Martino-IST, Genoa, Italy.

- National Institute for Health Research, UK.

Earlier drafts of this review were completed by the MORECare project, which was funded by the National Institute for Health Research and managed by the Medical Research Council as part of the Methodology Research Programme (project number: G0802654/1).

- The Atlantic Philanthropies and Cicely Saunders International, Other.

The review was finalised through support from The Atlantic Philanthropies and Cicely Saunders International. 